\title{
Olga Płaszczewska
}

Uniwersytet Jagielloński, Kraków

\section{LetTeratura itAliana e "ARTI SORElle" alla Facoltà DI LINGUA E LETTERATURA POLACCA: UN PERCORSO DI LETTERATURA COMPARATA}

Il corso di Letteratura italiana e "arti sorelle" è dedicato agli studenti di letteratura comparata presso la Facoltà di lingua e letteratura polacca all'Università Jagellonica di Cracovia. Si articola in 30 sedute di 90 minuti ciascuna, effettuate con cadenza settimanale, per complessive 60 ore di lezione. Nell'anno accademico 2012/2013 è giunto al terzo ciclo.

Obiettivo principale del corso è presentare ai partecipanti alcune opere di letteratura italiana sia nel loro contesto culturale, sia nell'ampia rete di relazioni con le più tradizionali arti visive (pittura, disegno e scultura) e con trasposizioni teatrali, cinematografiche e ambientazioni museali. Le lezioni tendono inoltre a sensibilizzare i partecipanti al problema della cosiddetta "corrispondenza delle arti" e delle sue realizzazioni storiche, presentandolo come uno degli spazi rilevanti della letteratura comparata.

Nonostante l'ambito letterario precipuamente italiano, le lezioni si svolgono in polacco, con ovvio ricorso a riferimenti e passaggi in originale. La tipologia dei partecipanti (di solito $35-45$ persone) è composita: date le modalità dei corsi per comparatisti, il gruppo comprende non soltanto rappresentanti di due livelli di studi polonistici (quello triennale di "laurea breve" e quello di laurea biennale, inclusi stranieri con borse di studio "Erasmus"), ma anche "ospiti" di altri indirizzi di studio filo- 
logici, fra i quali aspiranti italianisti. Questo comporta la necessità di adeguare argomenti e metodologia ad esigenze non omogenee: quelle di studenti che hanno una certa padronanza della lingua italiana e una più approfondita conoscenza della storia letteraria d'Italia (e quindi possono apprezzare testi e materiale audiovisivo in originale) e quelle di chi studia la letteratura italiana attraverso traduzioni recependola nel contesto della letteratura universale. La scelta dei testi è dunque in parte determinata dalle competenze linguistiche e culturali dei lettori. Ė perciò obbligatoria la lettura di testi tradotti in polacco mentre è facoltativa - ma ben accolta - la conoscenza dell'originale. La loro discussione richiede in ogni modo una breve presentazione di ciascun'opera sullo sfondo della "biografia artistica" dell'autore, arricchita di informazioni riguardanti la storia della ricezione del testo in Italia e in Europa. Questo elemento del corso assume funzione puramente indicativa, visto che oggetto primario delle lezioni non è la storia della letteratura italiana, ma le interazioni fra la letteratura e le "arti sorelle" (nel senso vasto del termine, a prescindere dal suo significato tradizionale, limitato alla sfera di pittura ed, eventualmente, musica $)^{1}$. Ciò nonostante, il corso è articolato secondo la cronologia letteraria, mentre l'esposizione delle situazioni artistiche correlabili segue il criterio tematico.

Le letture, scelte col proposito di suscitare la curiosità dello studente meno esperto d'italianistica, sono suddivise in due parti: la prima discussa durante il semestre invernale - comprende i testi dal Medioevo fino all'epoca della Controriforma; la seconda comporta esempi letterari del XIX e del XX secolo. La scelta dei testi è soggettiva, tuttavia si fonda in parte sui canoni della letteratura universale inclusi nel programma di studi di letteratura comparata, dove la Commedia di Dante e il Decameron di Boccaccio occupano una posizione significativa, mancano invece, se non per riferimenti, l'Orlando furioso di Ariosto e la Gerusa-

1 Cfr. E. Pantini, La letteratura e le altre arti, [in:] Letteratura comparata, (a cura di) A. Gnisci, Bruno Mondadori, Milano 2002, pp. 111-125; O. Płaszczewska, Przestrzenie komparatystyki-italianizm, Wydawnictwo Uniwersytetu Jagiellońskiego, Kraków 2010, pp. 213-236; C. Gibellini, Letteratura e arti figurative, [in:] Letteratura comparata, (a cura di) R. Bertazzoli, Editrice La Scuola, Brescia 2010, pp.158-190. 
lemme liberata di Tasso. Le letture otto- e novecentesche ${ }^{2}$ costituiscono un campo meno noto al generico lettore polacco, quindi richiedono un'introduzione leggermente più ampia rispetto ai "grandi classici" e un'accurata contestualizzazione storica. Per di più i testi vengono selezionati in base alle loro corrispondenze in campi d'arte, vale a dire in relazione allo sviluppo della tradizione iconografica, agli adattamenti cinematografici e alle interpretazioni musicali delle singoli opere.

Così della Commedia sono commentati i passaggi più frequentemente interpretati nelle arti visive e in musica: dall'Inferno, Dante nella selva oscura, l'episodio di Paolo e Francesca e quello del conte Ugolino, la selva dei suicidi con Pier della Vigna; dal Purgatorio, le vicende di Pia de' Tolomei e quelle di Sordello; dal Paradiso, la visione dell'Empireo e l'immagine di Beatrice. Nello stesso modo vengono scelti brani di letteratura più recente. Dai Promessi sposi, per esempio, si legge - per materiale storico e ricchezza iconografica del motivo - soltanto la storia della Monaca di Monza, esibita sullo sfondo dei riferimenti pittorici nell' 800 stesso, con accento sulla questione femminile, segnalata ormai durante le lezioni dedicate alle eroine dantesche, e con cenni a una novecentesca storia di Gertrude, che ritorna al suo vero nome di Marianna de Leyva nella pièce di Giovanni Testori messa in scena da Luchino Visconti.

Alcune considerazioni sulla fama di Manzoni e la sua ricezione in e fuori d'Italia aprono ad un discorso sul "culto dell'autore" nella civiltà europea in tutte le epoche. Nelle arti visive la venerazione dello scrittore si esprime in numerosi ritratti, realistici o immaginari, fra cui appare caratteristica la rappresentazione "sdoppiata" del soggetto, cioè ripetuta

2 Cioè I Promessi sposi di Alessandro Manzoni (trad. B. Sieroszewska); Piccolo mondo antico di Antonio Fogazzaro (trad. H. Kralowa), G. D'Annunzio, Il trionfo della morte (trad. L. Staff o S. Kasprzysiak), F. T. Marinetti e la poesia futurista (trad. J. Kurek), L. Pirandello, Enrico IV (trad. Z. Jachimecka), G. Tomasi di Lampedusa, Il Gattopardo (trad. Z. Ernstowa o S. Kasprzysiak); A. Moravia (un racconto da scegliere); E. de Filippo, Filumena Marturano (trad. J. Pasenkiewicz); I. Calvino, Se una notte d'inverno un viaggiatore (trad. A. Wasilewska); in più una scelta di poesia italiana (testi di G. Leopardi, G. Pascoli, G. Carducci, G. Ungaretti, A. Merini, L. Spaziani). 
nell'opera che ritrae, ad esempio, una statua che è di per sé un'immagine dell'autore ${ }^{3}$, oppure - come si osserva nel caso di Gabriele D'Annunzio, discusso in una lezione a parte - una fotografia che rappresenta lo scrittore mentre è ritrattato e la sua effigie in statu nascendi. Il discorso sulla corrispondenza delle arti entra qui nell'ambito della riflessione sul biografismo e l'autobiografismo letterario e i suoi aspetti extraletterari, tuttavia resta sempre strumento per avvicinare la cultura italiana allo studente polacco. La riflessione sull'autobiografismo dannunziano, basata sulla conoscenza delle opere del Vate e della sua vita, comprende anche il suo aspetto "museale", cioè il problema della creazione concepita anche come organizzazione artistica dello spazio, quindi un modo particolare di interferenze fra l'arte e la letteratura.

Generalmente, le interpretazioni artistiche di un'opera letteraria costituiscono il punto di partenza per un'analisi dettagliata dei suoi frammenti. Ad esempio, nelle lezioni riguardanti la Commedia, l'attenzione viene richiamata dapprima sulle modalità della lettura operata dal pittore/scultore oppure dal musicista. In questi casi l'interpretazione si concentra sulle affinità e sulle differenze fra l'esplicazione dantesca dell'episodio e la sua "proiezione" artistica. Il punto successivo della lettura è un'analisi degli aspetti tecnici ed espressivi, della stilistica propria dell'artista e del significato dell'opera nell'ambito della storia dell'arte. È chiaro che alcune delle realizzazioni sono discusse in particolar modo a causa del loro messaggio letterario piuttosto che per il loro valore artistico, comunque fra le realizzazioni citate non mancano nomi rilevanti, come quelli di Giotto, Sandro Botticelli, Jacopo Tintoretto, William Blake, Jean-Auguste-Dominique Ingres, Eugène Delacroix, Dante Gabriel Rossetti, William Holman Hunt, Auguste Rodin, Gaetano Previati, Alberto Savinio, Salvador Dalì.

I riferimenti pittorici alla letteratura vengono classificati secondo la loro tipologia "narrativa", quindi nella storia di Paolo e Francesca

3 Come il quadro di Emilio de Amenti, La lettura in famiglia di un punto commovente dei "Promessi sposi", 1876, Musei Civici, Pavia. Cfr. La monaca di Monza. La storia. La passione. Il processo, (a cura di) L. Tonani, Silvana Editoriale, Milano 2009, p. 163. 
(interpretata come una discussione filosofica fra Dante e Francesca sulla qualità del delitto, in cui il lettore viene sedotto, così come lo è Dante - personaggio, dall'eloquenza della donna che giustifica il peccato presentando l'adulterio come amore e, allo stesso tempo, come conseguenza della sua forza ineluttabile ${ }^{4}$ ) si distinguono le rappresentazioni della "lettura fatale", e così del punto culminante (il bacio degli innamorati), la morte dei due, le loro sorti postume, incluso l'incontro con Dante e Virgilio. Ciascuna di esse può assumere un valore illustrativo, tendente al realismo, o simbolico, come nelle realizzazioni di William Blake ${ }^{5}$. Le stesse frasi si sottomettono anche ad una catalogazione secondo l'interpretazione dell'amore di Francesca, dividendosi in quelle "giustificanti" (in cui viene sottolineata l'innocenza della donna, la sua passività nei confronti dell'amante, come accade nelle tele storicizzanti di Ingres ${ }^{6}$ ) e quelle "condannatorie" (in cui emerge la concupiscenza dei due, come nella soluzione attualizzante di Gaetano Previati). Per motivi didattici, invece, della produzione letteraria dei futuristi si discutono, a parte i manifesti, innanzitutto alcune poesie, perché permettono la lettura di un intero componimento. Si analizzano inoltre delle "tavole parolibere" (in riproduzioni proiettate sulla lavagna luminosa). Il discorso sulla poetica e sulla dottrina futurista è illustrato da riferimenti alle "opere di trasgressione", come metalliche Parole in libertà futuriste olfattive tattili-termiche o Litolatta, dove il limite fra la parola e l'immagine viene volontariamente eliminato per la sinestesia, creando una realtà d'espressione visiva ed intellettuale.

4 Cfr. P. Levine, Reforming the Humanities. Literature and Ethics from Dante through Modern Times, Palgrave Macmillan, New York - London 2009, pp. 3-7, 49-57; M. Cieśla-Korytowska, ,,Te książki zbójeckie...”, Wydawnictwo Uniwersytetu Jagiellońskiego, Kraków 2011, pp. 10-14, passim.

5 Cfr., fra l'altro, A. Konopacki, William Blake, Arkady, Warszawa 1987, tav. 28 con il commento; I. Ciseri, Il Romanticismo 1780-1860: la nascita di una nuova sensibilità, Mondadori Electa, Milano 2003, p. 252.

6 Cfr. fra l'altro, W. Hofmann, Une époque en rupture. 1750 - 1830, trad. M. Couffon, Gallimard, Paris 1995, pp. 633, 636-637; G. Vigne, Ingres, Citadelles \& Mazenod, Paris 1995, pp. 274 e passim. 
Mentre il discorso sulla pittura è illustrato attraverso una proiezione multimediale di riproduzioni delle opere discusse, la riflessione sulla musica è introdotta dall'ascolto di un passaggio, commentato prima e dopo l'audizione. L'ultima scena della Francesca da Rimini di Sergei Rachmaninov, analizzata nel suo ambito storico e musicale, conclude di solito il percorso di lettura del Canto V dell'Inferno, mentre l'Après une lecture du Dante di Ferenc Liszt permette un riepilogo del "filo dantesco" della corrispondenza delle arti. La musica quindi non necessariamente significa l'adattamento vero e proprio, né la trasmutazione di un componimento letterario in un'opera musicale. A volte l'accompagnamento sonoro, secondo la tradizione aulica, trasforma il testo poetico in una canzone autonoma. Il senso della parola e del suono risulta dal connubio fra le due forme d'espressione. Nel percorso didattico come illustrazione di questo tipo di corrispondenza serve, ad esempio, la messa in musica dell'episodio del battesimo di Clorinda della Gerusalemme liberata (XII, 66-68) proposta dal madrigalista Sigismondo d'India (c. 1582-1629), compositore non precisamente contemporaneo di Tasso, ma neanche da lui lontano culturalmente. Per il pubblico non sufficientemente "italianato" l'ascolto costituisce un'occasione propizia per ascoltare e "sentire" il testo in originale. Durante l'esecuzione del passaggio, il frammento cantato viene presentato su lavagna luminosa con la traduzione a fronte in modo da rendere possibile la comprensione e la ricezione emotiva dell'opera. La stessa strategia viene adoperata nel corso di lezioni dedicate alla lettura di alcuni esempi di poesia italiana.

Diverso è il caso degli adattamenti cinematografici della letteratura italiana. Durante il corso vengono discussi come una modalità non tradizionale di corrispondenze artistiche. Per esempio, il Decameron di Pier Paolo Pasolini è presentato nel campo di varie realizzazioni pittoriche sia della "cornice narrativa" dell'opera, sia delle singole novelle. Così l'episodio di Lisabetta da Messina (IV, 5) viene discusso prima in rapporto con interpretazioni letterarie della novella, come il poema di John Keats, Isabella, or the Pot of Basil, e delle sue realizzazioni iconografiche create nell'ambiente dei preraffaelliti, e dopo - nella versione filmica. Il sovrapporsi di riferimenti artistici e culturali nel film di Pasolini permette di sensibilizzare gli studenti sia all'orientamento po- 
litico e all'impegno sociale del regista, sia alla sua raffinatezza intellettuale e alla conoscenza profonda della realtà italiana. Può essere inoltre adoperato come illustrazione della continuità di interazioni fra le arti. Ad esempio, la trasformazione del Giudizio universale di Fra' Angelico e della Madonna in gloria di Giotto in una sorte di "quadro vivente" ripreso dal protagonista - pittore in cerca d'ispirazione artistica - viene letta sia come un'interpretazione dell'opera boccacciana, sia come un frutto d'ispirazione della pittura italiana, percepita come un elemento innato della mentalità popolare italiana. Lo stesso trasferimento degli eventi dall'ambiente aulico o borghese al mondo contadino permette di riflettere sulla libertà mentale del regista, che interpreta l'universo boccacciano in chiave della sua italianità primordiale, e sull'attualità della visione dell'uomo proposta da Boccaccio. Invece, la versione cinematografica del Piccolo mondo antico di Antonio Fogazzaro è esposta come l'unica interpretazione artistica del romanzo. Vengono analizzati i mezzi d'espressione tipici del calligrafismo cinematografico, fra cui la fedeltà ai particolari scenografici (dai costumi agli arredamenti degli spazi). Anche qui non mancano le citazioni culturali la cui esplicazione permette allo studente di capire meglio la civiltà italiana dell' 800 , sia nelle sue manifestazioni di costume, sia nell'eredità artistica "riprodotta" in codice filmico (sono caratteristiche le citazioni di paesaggi di Como dalla pittura ottocentesca lombarda ${ }^{7}$ oppure quelle di vestiario infantile copiato da ritratti di Francesco $\mathrm{Hayez}^{8}$, e canti patriottici nella colonna sonora del film). Sui modi d'interpretazione del romanzo (fra cui le operazioni di riduzione e ampliamento) si fonda la riflessione sul Gattopardo portato sullo schermo da Luchino Visconti (1963). Si nota dunque che il rispetto del dettaglio storico rende conforme il film al

7 Per esempio: M. D’Azeglio, Veduta del lago di Como, ramo di Lecco (1831), coll. privata, Milano; G. Canella, Plenilunio (1840), Galleria d'Arte Moderna e Contemporanea, Brescia. Cfr. I. Ciseri, op. cit., pp. 291, 293.

8 Per esempio, F. Hayez, Ritratto della contessina Luigia Negroni Prati Morosini bambina (1867), coll. privata (cartolina Marsilio Edizioni, dalla mostra Hayez. Dal mito al bacio, Palazzo Zabarella, Padova 1998-1999); F. Hayez, Ritratto della contessina Antonietta Negroni Prati Morosini bambina (1858). Cfr. F. Mazzocca, Hayez, „Art \& Dossier” 137 (settembre 1998), p. 33. 
messaggio ideologico del romanzo. L'esposizione dell'argomento comprende anche un'approfondita analisi dei temi melodici ricorrenti nel film come strumenti di costruzione del "mondo rappresentato". Invece la lettura della novella Troppo ricca di Alberto Moravia, confrontata con l'episodio Anna di Ieri, oggi, domani (1963) del regista Vittorio De Sica, apre la discussione sull'efficacia espressiva della parola e dell'immagine. Grazie alla maestria delle creazioni di Sophia Loren e di Marcello Mastroianni diventa possibile porre in discussione le convinzioni circa il primato espressivo della letteratura sul cinema, perché nel raffronto col film di De Sica, in cui si gioca sulle diverse sfumature delle relazioni interpersonali, la novella di Moravia, pur altrettanto moralista come il suo adattamento cinematografico, risulta piuttosto scialba.

Leggermente diverso è l'approccio metodologico nei confronti del teatro italiano, rappresentato nel corso da due pièce: Enrico IV di Luigi Pirandello e Filumena Marturano di Eduardo de Filippo. L'opera di Pirandello viene letta come un testo esemplare in cui la questione dell'immagine, identificabile con l'apparenza e fonte di illusioni, svolge un ruolo significativo. L'attenzione del lettore si concentra sul quadro (ritratto reale) intorno al quale si sviluppa l'intreccio in cui fingere significa non soltanto recitare se si sta in un contesto storico traslato, ma anche travestirsi da se stessi "perpetuati" nel ritratto fittizio, rappresentante una figura mascherata. Nella riflessione sulla corrispondenza delle arti, la lettura del dramma pirandelliano presenta l'artefatto come un oggetto dell'elaborazione artistica, dell'interpretazione interna ("l'arte nell'arte" nella dimensione più ampia di quella scenografica, perché profondamente correlata all'esistenza dell'uomo). Invece l'amara commedia di De Filippo, presentata insieme alla biografia artistica dell'autore e letta in una traduzione teatrale, in Polonia mai stampata ma resa pubblica attraverso messe in scena a Łodź, a Varsavia e a Cracovia, presso il Teatro Giulio Słowacki", viene confrontata con il "libero adattamento" proposto da Vittorio De Sica nel suo Matrimonio all'italiana.

9 E. De Filippo, Filumena Marturano, trad. J. Pasenkiewicz, (1967?), un copione teatrale gentilmente concesso all'uso dei corsisti dalla Direzione dell'archivio del Teatro Słowacki. 
Torna dunque la questione del "trasferimento" della materia teatrale al cinema, dei loro strumenti d'espressione corrispondenti o contrastanti, del ruolo del regista come mediatore del testo letterario, e della valenza interpretativa degli attori principali (ancora Loren e Mastroianni) che di per sé costituisce un confronto con le versioni precedenti (sia teatrali, sia filmiche, come quella del 1951 con la partecipazione di Titina De Filippo e dello stesso Eduardo).

Durante lezioni dedicate al film e al teatro lo studente ha la possibilità di guardare in originale una selezione di scene dei film trattati (l'episodio Anna di Ieri, oggi, domani, in virtù della sua concisione, è citato interamente), con una traduzione simultanea del docente e/o con il dialogato tradotto in versione cartacea. Grazie alla precedente conoscenza del testo letterario di partenza, il ricorso alla lingua originale non ostacola la percezione del filmato da parte degli studenti, anzi, li invoglia allo studio dell'italiano o al perfezionamento delle loro competenze linguistiche assieme a quelle culturali.

Vista la vastità degli argomenti che richiedono varie metodologie didattiche e percettive, il corso non è di semplice attuazione né dal punto di vista del docente né da quello dello studente. Una profonda erudizione artistica, comprendente vasti campi di storia e critica dell'arte, del cinema, del teatro e della musica, la capacità di padroneggiare temi e letterature di diverse epoche, insieme alla preparazione filologica sono requisiti necessari per il docente. Oltre che dalla preparazione di chi lo conduce, la qualità del corso è determinata anche dalle possibilità tecniche dell'Università. Per un appropriato ed efficace percorso d'insegnamento è necessaria un'aula fornita di lavagna luminosa (o di proiettore multimediale) con una postazione computer per la presentazione delle riproduzioni e altri contenuti visivi, di un impianto audio per l'ascolto dei brani musicali e di un'apparecchiatura video (dvd) per la proiezione dei filmati.

Una precisazione va fatta circa la disponibilità di un manuale della materia, strumento molto richiesto dai partecipanti al corso sin dalla prima lezione. Il manuale, come spesso accade nel campo della letteratura comparata, che è una disciplina fondata sulla prassi interpretativa e quindi naturalmente contraria ad ogni tipo di teoretizzazione del di- 
scorso $^{10}$, inevitabile per un compendio accademico, semplicemente non esiste. Le lezioni in aula costituiscono dunque la fonte e insieme l'occasione primaria di formazione e informazione. I partecipanti sono perciò fermamente invitati a ridurre al minimo le eventuali assenze.

La prova finale consiste in un test scritto a domande aperte, riguardanti sia la conoscenza delle letture indicate (è richiesta la lettura dei frammenti stabiliti delle opere più antiche, da Dante a Tasso, e la conoscenza di testi dell'Otto- e Novecento completi, mentre durante le lezioni si discutono i loro passaggi rappresentativi in relazione all'argomento trattato), sia i contenuti del corso intero. Fra vari compiti inclusi nel test c'è sempre il riconoscimento di un'opera visuale (un quadro, una grafica, una statua, ecc.) nel suo contesto letterario e culturale nonché l'interpretazione di un passaggio del testo e l'indicazione dei suoi riferimenti artistici. Questa prova è obbligatoria per tutti. Chi ne abbia interesse può sostenere l'esame (orale) sulla conoscenza approfondita degli argomenti trattati durante il corso, aggiungendo una riflessione su un altro caso di corrispondenza fra un testo scelto dallo studente (previa approvazione della docente) e l'arte.

Considerate le competenze del gruppo, eterogeneo dal punto di vista linguistico e formativo, in relazione ai vari livelli di conoscenza della cultura italiana e dell'arte europea, all'inizio del corso si indicano - come ausiliari - alcuni suggerimenti bibliografici fra cui lo studente può scegliere prontuari e vademecum a suo giudizio. Durante il corso quest'elenco generico viene completato con indicazioni più specifiche, distribuite attraverso la piattaforma multimediale „Pegaz,” indirizzate ai partecipanti particolarmente interessati ad un dato argomento. La lista di suggerimenti bibliografici di base comprende dunque manuali tradizionali di storia della letteratura italiana in polacco ${ }^{11} \mathrm{e}$ in italiano ${ }^{12}$. Come

10 Cfr. O. Płaszczewska, op. cit., pp. 298-299.

11 Per esempio, K. Żaboklicki, Historia literatury włoskiej, PWN, Warszawa 2008 e Historia literatury wtoskiej XX wieku, (a cura di) J. Ugniewska, PWN, Warszawa 2001.

12 Ad esempio, G. Ferroni, Profilo storico della letteratura italiana, Einaudi scuola, Milano 1999 e Storia della letteratura italiana, vol. 1-9, (a cura di) E. Cecchi, N. Sapegno, Garzanti, Milano 1969. 
prontuario di consultazione rapida può anche servire una pubblicazione certo datata, ma non priva di attualità: il dizionario di letteratura italiana a cura di Mieczysław Brahmer ${ }^{13}$. Inoltre, fra i compendi suggeriti si trovano due volumi della storia del teatro in Italia, editi dal team di italianiste cracoviane ${ }^{14}$. Per quanto riguarda il cinema, lo studente ha a disposizione sia la monografia di Tadeusz Miczka ${ }^{15}$, sia il volumetto dedicato alla letteratura italiana appena uscito nella collana "Literatura na ekranie" [Letteratura sullo schermo] ${ }^{16}$. Per un minimo orientamento nel largo spazio della corrispondenza delle arti vengono proposti i popolari “Dizionari dell'Arte” dell'Electa (in Polonia editi presso l'Arkady), fra cui si consigliano soprattutto Episodi e personaggi della letteratura di Francesca Pellegrino (2003) ${ }^{17}$, sia alcuni manuali di storia dell'arte, accessibili nelle biblioteche di Cracovia ${ }^{18}$ e vari compendi musicali ${ }^{19}$.

$\mathrm{Ci}$ si attende che a conclusione del corso lo studente pervenga a una soddisfacente conoscenza di base della letteratura italiana nel suo contesto europeo. Dovrebbe quindi conoscere i più significativi rappresentanti della civiltà italiana di varie epoche letterarie e artistiche. In più dovrebbe distinguere i principali percorsi della storia della letteratura

13 Maty stownik pisarzy wtoskich, (a cura di) M. Brahmer e al., Wiedza Powszechna, Warszawa 1969.

14 M. Gurgul, J. Miszalska, M. Surma-Gawłowska, Historia teatru i dramatu włoskiego, voll. 1-2, Universitas, Kraków 2008.

15 T. Miczka, Kino włoskie, słowo/obraz/terytoria, Gdańsk 2009.

16 Od Boccaccia do Tabucchiego. Adaptacje literatury włoskiej, (a cura di) A. Gałkowski, A. Miller-Klejsa, Warszawa 2012.

17 F. Pellegrino, Literatura. Postacie i watki, trad. T. Łozińska, Arkady, Warszawa 2008

18 A partire dalla monografia di E. H. Gombrich, O sztuce, [traduttori vari], Rebis, Poznań 2008, e dall'ampio saggio di Jan Białostocki, Sztuka cenniejsza niż złoto, PWN, Warszawa 2001; H. W. Janson, History of Art (1962), voll. 1-2, H. N. Abrams, New York 1995; A. Chastel, Sztuka włoska, trad. E. Bąkowska, voll. 1-2, Wydawnictwa Artystyczne i Filmowe, Warszawa 1978; fino a quella di Mieczysław Porębski, Dzieje sztuki w zarysie, voll. 1-3, Arkady, Warszawa 1976-1988.

19 Come P. Kamiński, Tysią i jedna opera, voll. 1-2, PWM, Kraków 2008; I. Turska, Przewodnik baletowy, PWM, Kraków 1989; T. Chylińska, S. Haraschin, B. Schaeffer, Przewodnik koncertowy, PWM, Kraków 1991. 
italiana e saper non soltanto confrontarla con la storia della letteratura polacca, ma anche interpretarla in una prospettiva universale. Dovrebbe essere consapevole delle convenzioni letterarie e capace di indicare i capisaldi della letteratura italiana insieme alle loro interpretazioni nella cultura europea, dal medioevo ai tempi moderni. Inoltre, lo studente dovrebbe disporre di elementari nozioni sulle relazioni fra letteratura e pittura, letteratura e arti visive, letteratura e musica. Dovrebbe orientarsi nella principale metodologia dell'interpretazione e dell'analisi di testi letterari, opere d'arte e musica nell'ambito della letteratura comparata, così come saper interpretare un testo, collocandolo nel contesto storico, culturale e biografico. Dovrebbe essere in grado di trovare riferimenti fra un testo letterario e opere d'arte non espresse in parola. Per di più, lo studente del corso di Letteratura italiana e "arti sorelle" dovrebbe riuscire ad analizzare e paragonare testi in polacco e italiano, seguire le interferenze genetiche e tipologiche fra la letteratura e le arti. Dovrebbe, in conclusione, maturare la consapevolezza del significato della letteratura e delle civiltà italiane per la letteratura e cultura polacca ed europea, sapendo valutare il loro ruolo nella formazione dell'eredità culturale del mondo. Innanzitutto, si spera che scopra, in un modo o nell'altro, il piacere di "convivere" con la letteratura italiana e che trovi quindi efficaci stimoli per indagare i segreti dell'arte europea e dei suoi legami con la letteratura d'ogni tempo.

\section{Italian literature and the "Sister Arts" at the Faculty OF Polish Studies: A COURSE IN COMPARATIVe literature}

\section{Summary}

The purpose of the article is to outline the guidelines of the interdisciplinary course in comparative literature, given at the Jagiellonian University in Krakow. The subject of the series of lectures is the relation between Italian literature and different branches of art, in context of their cultural references. The strategies of sensibilization of the audience both to the problem of ,sister arts" and to the Italian literature and language themselves are explained. In addition, the significance of an interdisciplinary approach to the problem of reception of Italian literature in European culture is mentioned as one 
of the aims of the lecture. Moreover, main problems of the course, such as themes of reintepretation of Italian classics in visual arts, music, cinema and theatre, are discussed on the background of „classroom dynamics” which characterize the group of students with different knowledge of Italian language and different cultural competences.

Keywords: comparative literature, sister arts, transmutation, Italian literature and culture in modern art, reception of Italian literature in Poland and in Europe, teaching Italian literature in its artistic context 